\title{
Which is more important, xanthine oxidase activity or uric acid itself, in the risk for cardiovascular disease?
}

\author{
Takuya Tsuchihashi ${ }^{1}$
}

Received: 9 July 2021 / Revised: 13 July 2021 / Accepted: 14 July 2021 / Published online: 18 August 2021

(C) The Japanese Society of Hypertension 2021

The prevalence of gout and asymptomatic hyperuricemia, defined as a serum uric acid (UA) level $>7.0 \mathrm{mg} / \mathrm{dL}$, is increasing in Japan [1]. Both genetically determined factors that promote the production of UA or reduce its excretion and various environmental factors contribute to the development of hyperuricemia. Asymptomatic hyperuricemia is frequently associated with lifestyle-related diseases such as hypertension, diabetes mellitus, and metabolic syndrome. These diseases are known to be associated with insulin resistance. Hyperinsulinemia caused by insulin resistance facilitates the reabsorption of UA by urate transporter 1 (URAT1), which localizes to the luminal side of renal proximal tubular cells, leading to the increase in serum UA. Hyperuricemia is also frequently associated with chronic kidney disease (CKD) and cardiovascular disease. However, it remains unclear whether hyperuricemia is causally related to the development of these diseases, because the relationship between UA and other cardiovascular risk factors shows considerable potential for confounding and reverse causation. Indeed, a previous report utilizing systematic reviews, meta-analyses, and Mendelian randomization studies showed that convincing evidence of a clear role for serum UA level exists only for gout and nephrolithiasis [2]. On the other hand, recent Mendelian randomization analyses showed that the increase in genetically predicted serum UA was associated with increased risks of coronary heart disease, peripheral artery disease, and stroke [3]. Genetically predicted serum UA was also associated with an increase in blood pressure (BP), and elevated BP was estimated to mediate approximately one-third of the effect of UA on cardiovascular disease risk [3]. Furthermore, a systematic review and meta-analysis of randomized controlled trials (RCTs) showed a favorable effect of urate-lowering

Takuya Tsuchihashi

tuti@ns.yawata-mhp.or.jp

1 Steel Memorial Yawata Hospital, Kitakyushu City, Japan treatment on BP. Such treatment also reduced major adverse cardiovascular events (cardiovascular death, nonfatal myocardial infarction, unstable angina requiring urgent revascularization, or nonfatal stroke) in the subjects with previous cardiovascular disease [3]. Taken together, Mendelian randomization analyses and clinical trial data suggest that higher serum UA increases BP, which may in turn raise cardiovascular disease risk. In addition, urate-lowering treatment seems beneficial for lowering BP and reducing cardiovascular events, at least in high-risk subjects. In the 3rd edition of the Japanese guidelines on the management of hyperuricemia and gout published in 2018, the rationality of urate-lowering treatment was evaluated [1]. Beneficial outcomes were found, so urate-lowering agents are recommended in hyperuricemic patients with CKD, while uratelowering agents cannot be recommended for the purpose of improving prognosis in hyperuricemic patients with either hypertension or heart failure. Further studies are required to verify the rationality of pharmacological treatment of asymptomatic hyperuricemic patients with hypertension, CKD or other cardiovascular diseases.

Accumulating evidence indicates that xanthine oxidase (XO) activity rather than UA itself has a major role in tissue injury and target organ damage. Xanthine oxidoreductase (XOR) has a major role in the formation of UA (Fig. 1). $\mathrm{XOR}$ is composed of xanthine dehydrogenase $(\mathrm{XDH})$ and $\mathrm{XO}$. XO is generated from XDH by posttranslational modification and catalyzes the last 2 steps of reactions that convert hypoxanthine to xanthine and xanthine to UA. These reactions yield superoxide and hydrogen peroxide. Therefore, activation of XO can be a source of reactive oxygen species, leading to tissue injury. Circulating XO can bind to vascular cells and interact directly with $\mathrm{NO}$, leading to a decrease in NO and an increase in peroxynitrite. On the other hand, serum UA has been reported to act on vascular smooth muscle cells or endothelial cells via uric acid transporters (UATs). When taken up by endothelial cells, UA reduces the level of NO by inhibiting its production and accelerating its degradation. URAT1 is specifically 


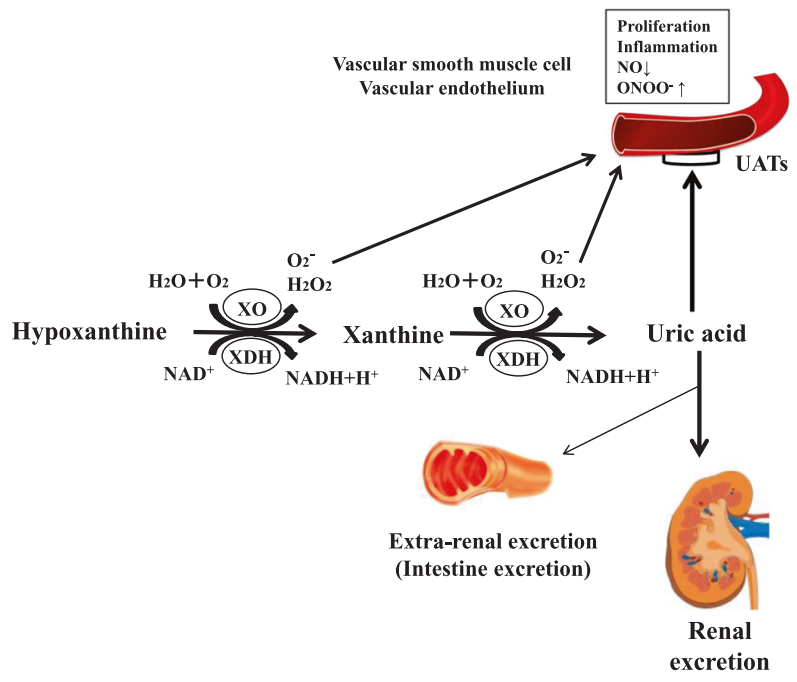

Fig. 1 Schematic representation of the mechanism of vascular injury caused by xanthine oxidase activation or uric acid itself. XO xanthine oxidase, XDH xanthine dehydrogenase, UATs uric acid transporters

expressed on human vascular smooth muscle cells, providing a mechanism by which UA enters human vascular smooth muscle cells [4]. Since UA-induced human vascular smooth muscle cell proliferation and the decrease in NO in human umbilical vein endothelial cells (HUVECs) are inhibited by the organic anion transport inhibitor probenecid, the effect of UA seems to be exerted via UATs [5]. These observations raise the question of which is more important, the inhibition of XO activity or the decrease in serum UA, in protecting against cardiovascular and other tissue damage. In the current issue of Hypertension Research, Furuhashi et al. report that plasma XO activity was independently associated with hypertension in nondiabetic individuals of the general population, speculating that the activation of XO may promote oxidative stress-related injury to endothelial cells and the kidney, leading to the elevation of BP [6]. Another report showing that $\mathrm{XO}$ genetic polymorphisms predict changes in $\mathrm{BP}$ and the risk of hypertension supports the crucial role of $\mathrm{XO}$ in the incidence of hypertension [7]. Regarding endothelial function in the clinical setting, $\mathrm{XO}$ activity is reported to correlate with vascular endothelial dysfunction in patients with type 1 diabetes mellitus [8]. The observation that a high dose of the XO inhibitor allopurinol but not the uricosuric agent probenecid improved endothelial function in patients with chronic heart failure suggests that reducing vascular oxidative stress but not lowering UA is important for improving endothelial function [9]. XO activity was also an independent predictor of cardiovascular events in CKD and hemodialysis patients, regardless of the UA level, suggesting a role for XO activity in CKD-related cardiovascular disease [10]. Contrary to these observations, an RCT comparing the effects of allopurinol and probenecid showed similar BP reductions in hyperuricemic adolescents, which does not support a superior role of $\mathrm{XO}$ inhibition to the reduction of serum $\mathrm{UA}$, at least in the aspect of $\mathrm{BP}$ reduction [11].

XO inhibitors (UA production-inhibitory drugs) and UAT inhibitors (uricosuric agents) are selected for treating hyperuricemia classified as the UA-overproductive type and the UA-underexcretory type, respectively [1]. Since most patients with hypertension or CKD show an underexcretory type of hyperuricemia, it seems reasonable to use uricosuric agents. If the inhibition of $\mathrm{XO}$ activity rather than the decrease in serum UA is important for the prevention of tissue or target organ damage, the use of XO inhibitors might be recommended. To address this issue, a randomized crossover trial comparing a standard dose of the XO inhibitor febuxostat, the uricosuric agent benzbromarone, and a low-dose combination of both drugs was examined in a small number of hypertensive patients with hyperuricemia [12]. As a result, the decrease in UA was significantly greater with a low-dose combination therapy than with a standard dose of each drug alone. Endothelial function evaluated by flow-mediated vasodilatation was not significantly different between the phases when the patients took febuxostat and benzbromarone alone. This finding does not support a superior role of XO inhibition in the improvement of endothelial function in hypertensive patients. Whether the preferential use of XO inhibitors becomes a new therapeutic strategy for the prevention of cardiovascular disease in patients with asymptomatic hyperuricemia awaits further high-quality trials.

\section{Compliance with ethical standards}

Conflict of interest The author received honoraria from Takeda Pharmaceutical Co., Limited, Daiichi Sankyo Co., Limited, and Teijin Pharma Limited.

Publisher's note Springer Nature remains neutral with regard to jurisdictional claims in published maps and institutional affiliations.

\section{References}

1. Hisatome I, Ichida K, Mineo I, Ohtahara A, Ogino K, Kuwahara M, et al. Japanese Society of Gout and Uric \& Nucleic Acids 2019 Guidelines for Management of Hyperuricemia and Gout 3rd edition. Gout Uric Nucleic Acids. 2020;44:sp-1-sp-40.

2. Li X, Meng X, Timofeeva M, Tzoulaki I, Tsilidis KK, Ioannidis JP, et al. Serum uric acid levels and multiple health outcomes: umbrella review of evidence from observational studies, randomized controlled trials, and Mendelian randomisation studies. BMJ 2017;357:j2376.

3. Gill DG, Cameron AC, Burgess S, Li X, Doherty DJ, Karhunen $\mathrm{V}$, et al. Urate, blood pressure, and cardiovascular disease. Enidence from Mendelian randomization and meta-analysis of clinical trials. Hypertension. 2021;77:383-92.

4. Price KL, Sautin Y, Long DA, Zhang L, Miyazaki H, Mu W, et al. Human vascular smooth muscle cells express a urate transporter. J Am Soc Nephrol. 2006;17:1791-5. 
5. Kang DH, Park SK, Lee IK, Johnson RJ. Uric acid-induced Creactive protein expression: Implication of cell proliferation and nitric oxide production of human vascular cells. J Am Soc Nephrol. 2005;16:3553-62.

6. Furuhashi M, Higashiura Y, Koyama M, Tanaka M, Murase T, Nakamura $\mathrm{T}$, et al. Independent association of plasma xanthine oxidoreductase activity with hypertension in nondiabetic subjects not using medication. Hypertens Res. https://doi.org/10.1038/ s41440-021-00679-1 2021.

7. Scheepers LEJM, Wei FF, Stolarz-Skrzypek K, Malyutina S, Tikhonoff V, Thijs L, et al. Xanthine oxidase gene variants and their association with blood pressure and incident hypertension: a population study. J Hypertens. 2016;34:2147-54.

8. Washio K, Kusunoki Y, Tsunoda T, Osugi K, Ohigashi M, Murase $\mathrm{T}$, et al. Xanthine oxidoreductase activity correlates with vascular endothelial dysfunction in patients with type 1 diabetes. Acta Diabetologica. 2020;57:31-39.

9. George J, Carr E, Davies J, Belch JJF, Struthers A. High-dose allopurinol improves endothelial function by profoundly reducing vascular oxidative stress and not by lowering uric acid. Circulation. 2006;114:2508-16.

10. Gondouin B, Jpoude-Chiche N, Sallee M, Dou L, Cerini C, Loundou A, et al. Plasma xanthine oxidase activity is predictive of cardiovascular disease in patients with chronic kidney disease, independently of uric acid levels. Nephron. 2015;13:167-74.

11. Soletsky B, Feig DI. Uric acid reduction rectifies prehypertension in obese adolescents. Hypertension. 2012;60:1148-56.

12. Ohta Y, Ishizuka A, Arima H, Hayashi S, Iwashima Y, Kishida M, et al. Effective uric acid-lowering treatment for hypertensive patients with hyperuricemia. Hypertens Res. 2016;40:259-63. 